\title{
How to Compute Spectra with Error Control
}

\author{
Matthew J. Colbrook, Bogdan Roman, and Anders C. Hansen \\ Department of Applied Mathematics and Theoretical Physics, University of Cambridge, \\ Wilberforce Road, Cambridge CB3 OWA, United Kingdom
}

(Received 28 November 2018; published 28 June 2019)

\begin{abstract}
Computing the spectra of operators is a fundamental problem in the sciences, with wide-ranging applications in condensed-matter physics, quantum mechanics and chemistry, statistical mechanics, etc. While there are algorithms that in certain cases converge to the spectrum, no general procedure is known that (a) always converges, (b) provides bounds on the errors of approximation, and (c) provides approximate eigenvectors. This may lead to incorrect simulations. It has been an open problem since the 1950s to decide whether such reliable methods exist at all. We affirmatively resolve this question, and the algorithms provided are optimal, realizing the boundary of what digital computers can achieve. Moreover, they are easy to implement and parallelize, offer fundamental speed-ups, and allow problems that before, regardless of computing power, were out of reach. Results are demonstrated on difficult problems such as the spectra of quasicrystals and non-Hermitian phase transitions in optics.
\end{abstract}

DOI: 10.1103/PhysRevLett.122.250201

Introduction.-It is hard to overestimate the importance of computing the spectra of operators in mathematical physics, quantum chemistry, condensed-matter physics, statistical mechanics, Hermitian, as well as non-Hermitian, quantum mechanics, quasicrystals, optics, and many other fields. Motivated by the many applications, the topic has been intensely investigated, by both physicists [1-9] and mathematicians [10-17], since the 1950s. A reliable algorithm should converge and guarantee that any point of the output is close to the spectrum, up to a chosen arbitrary small error tolerance. A key question is whether such algorithms exist. Despite more than 90 years of quantum theory, the answer to this question has been unknown, even for Schrödinger operators.

The importance of this question is highlighted by the current interest in the spectral properties of systems with complicated spectra. The study of aperiodic systems, such as quasicrystals $[18,19]$, often leads to complicated, even fractal-like spectra [20-24], which can make current methods of computation difficult. Another example is given by recent experimental breakthroughs in open systems in optics, which typically yield non-Hermitian Hamiltonians as there is no guaranteed energy preservation [25-29]. No previously known algorithm can handle nonHermitian operators.

Questions on the foundations of computation and spectral computations have a rich history in physics. A recent example is the proof of the undecidability of the spectral gap [30]. Namely, one cannot construct an algorithm to determine whether a translationally invariant spin lattice system is gapped or gapless in the thermodynamic limit, a surprising result connected to seminal results in condensedmatter theory [31-33]. Hence, there are limitations to what a computer can achieve regarding limits of finite-dimensional systems.

In this Letter, we establish the boundaries for spectral problems in infinite dimensions. We show that it is impossible to design an algorithm for computing the spectra of Schrödinger operators which, given $\epsilon>0$, halts and produces an output that is $\epsilon$ away from the true spectrum as measured in the Hausdorff metric. In other words, using information from a finite patch (truncation) of an operator $A$, it is impossible to produce an approximation $\Gamma(A)$ to the spectrum $\operatorname{Sp}(A)$, which satisfies the two inequalities (I) $\operatorname{dist}(z, \operatorname{Sp}(A)) \leq \epsilon$, for all $z \in \Gamma(A)$, and also (II) $\operatorname{dist}(w, \Gamma(A)) \leq \epsilon$, for all $w \in \operatorname{Sp}(A)$, simultaneously. However, we show that it is possible to create approximations, converging to the spectrum, that satisfy inequality (I). Indeed, we know the approximation is sound or reliable, but we do not know if we have got everything yet.

Namely, we provide an algorithm $\Gamma_{n}(\cdot)$, which both converges to the spectrum $\operatorname{Sp}(A)$ in the Hausdorff metric as $n \rightarrow \infty$ and also computes a local error bound function $E(n, z)$. Here, $n$ is the size of the truncation of $A$ used to compute $\Gamma_{n}(A)$, whereas $z \in \Gamma_{n}(A)$ approximates a member of $\operatorname{Sp}(A)$. For discrete lattice operators, $n$ corresponds to considering the first $n$ basis sites and their interactions, whereas, in general, $n$ corresponds to the size of the truncation with respect to an orthonormal basis of the Hilbert space. The function $E(n, z)$ [defined in Eq. (4) in Ref. [34]] bounds the error of the approximations $z$ through the inequality $\operatorname{dist}(z, \operatorname{Sp}(A)) \leq E(n, z)$. Moreover, we have

$$
E_{n}(A):=\sup _{z \in \Gamma_{n}(A)} E(n, z) \rightarrow 0, \quad n \rightarrow \infty .
$$


This means that $\Gamma_{n}(A)$ is contained in the true bulk spectrum of $A$ up to the bound $E_{n}(A)$, regardless of how $A$ extends outside the patch of size $n$ used to compute $\Gamma_{n}(A)$. This is made possible through approximating the resolvent norm of $A$ via using certain folded Hamiltonians, as outlined in the methods section.

The algorithm is applied to several problems known to be difficult in the literature and can also compute approximate states. The new algorithm has another key advantage over current methods-it is entirely local or parallelizable.

Statement of the results.-Consider a lattice model $G$ with countably many vertices $V(G)$ (e.g., $\mathbb{Z}^{d}$ ) and write $v \sim_{k} w$ for $v, w \in V$ if there is a path of at most $k$ edges connecting $v$ and $w$. Let $\Omega_{G}$ be the class of normal finite range interaction Hamiltonians on $V(G)$, which are operators of the form

$$
A=\sum_{v \sim{ }_{k} w} \alpha(v, w)|v\rangle\langle w|
$$

for some $k$ (for more general operators, see [34]). The new algorithm computes $\mathrm{Sp}(A)$ and provides approximate eigenvectors (states). More precisely, (i) we prove that, given $\epsilon>0$, there does not exist any algorithm $\Gamma$ with input $A \in \Omega_{G}$ that halts and produces an output $\Gamma(A)$ with $d_{H}[\Gamma(A), \operatorname{Sp}(A)] \leq \epsilon$, where $d_{H}$ denotes the Hausdorff metric. (ii) However, we provide an algorithm, $\Gamma_{n}(\cdot)$, that uses only the matrix elements $\alpha(v, w)$, which converges in the Hausdorff metric to $\operatorname{Sp}(A)$ for any $A \in \Omega_{G}$ as $n \rightarrow \infty$ and such that the local error bound function $E(n, z)$ satisfies $\operatorname{dist}(z, \operatorname{Sp}(A)) \leq E(n, z)$ with $E_{n}(A) \rightarrow 0$.

This has direct implications in computational boundaries in quantum mechanics [34]. With a minor modification, the algorithm also computes the pseudospectrum [15,42], $\operatorname{Sp}_{\epsilon}(A)=\left\{z:\left\|(A-z I)^{-1}\right\|^{-1} \leq \epsilon\right\}$, a generalization of the spectrum (and measure of its stability) which is popular for non-Hermitian problems, for which the above are still true. All cases of our algorithm(s) share several key features: (i) Sharpness.-They realize the boundary of what digital computers can achieve. (ii) Known error of the output.-The error bound $E_{n}(A)$ is computed with no added complexity. (iii) Local computation of the spectrum.-Computations are local and can be restricted to regions such as the extreme parts or parts in the middle of the spectrum (excited states). The algorithm can efficiently compute states corresponding to any chosen part of the output without diagonalizing the whole matrix. (iv) Parallelization and speed.-Local computation immediately implies parallelization. Every area where one wants to compute the spectrum can be divided into suitable subsets that can be handled individually. This allows for a substantial speed-up given access to several computer cores.

Methods.-The main ideas of the algorithm for computing the spectrum of $A \in \Omega_{G}$ are as follows (see [34] for generalizations, non-normal operators, proofs, and pseudocodes). By ordering the vertices or sites, we can consider $A$ as an infinite matrix acting on $l^{2}(\mathbb{N})$, the space of square summable sequences. Given an integer $n$, consider the first $n$ basis vectors $\left|e_{1}\right\rangle, \ldots,\left|e_{n}\right\rangle$ and let $f(n)$ be minimal such that $\left\langle e_{i}|A| e_{j}\right\rangle=0$ if $j=1, \ldots, n$ and $i>f(n)$. Such a $f(n)$ exists by the assumption of the finite range of interactions. We then consider the rectangular matrix $P_{f(n)} A P_{n}$, where $P_{n}$ denotes orthogonal projection onto the span of the first $n$ basis vectors. Physically, the rectangular matrix $P_{f(n)} A P_{n}$ contains all of the interactions of the first $n$ sites without needing to apply boundary conditions (the range of the interactions controls precisely how rectangular the matrix should be).

The error bound function is then given as

$$
E(n, z) \approx \min \left\{\|(A-z I) x\|: x \in \operatorname{span}\left\{\left|e_{1}\right\rangle, \ldots,\left|e_{n}\right\rangle\right\}\right\},
$$

and we provide an efficient routine for its computation. This corresponds to an estimate of the distance of $z$ to the spectrum and physically corresponds to approximating the square root of the ground state energy of the folded Hamiltonian $P_{n}(A-z I)^{*}(A-z I) P_{n}$ on the domain $\operatorname{span}\left\{\left|e_{1}\right\rangle, \ldots,\left|e_{n}\right\rangle\right\}$. We prove that our approximation converges uniformly to the resolvent norm $\left\|(A-z I)^{-1}\right\|^{-1}=\operatorname{dist}(z, \operatorname{Sp}(A))$, on compact subsets of the complex plane. The convergence is also from above, meaning that we gain the rigorous error bound $\operatorname{dist}(z, \operatorname{Sp}(A)) \leq E(n, z)$. It is precisely the use of the rectangular truncation $P_{f(n)} A P_{n}$ that leads to convergence from above, and, in general, taking a square truncation will not even converge.

Given a region $\mathcal{D} \subset \mathbb{C}$ of interest, the other ingredient of the algorithm is a search routine that seeks to approximate the spectrum locally on $\mathcal{D}$. We consider a grid of points $G_{\mathcal{D}}(n)$ of spacing $\delta(n) \rightarrow 0$ as $n \rightarrow \infty$. The resolution $\delta(n)^{-1}$ (which can be viewed as a discretization parameter) can be changed to allow one to vary the number of computed solutions. In our experiments, we chose $\delta(n)$ to ensure $\approx n$ solutions for fair comparisons with other methods. The first step is to compute $E(n, \cdot)$ over $G_{\mathcal{D}}(n)$, which can be done in parallel. Given $z \in G_{\mathcal{D}}(n)$, we let $I_{z}$ be the points in $G_{\mathcal{D}}(n)$ at a distance most $E(n, z)$ away from $z$. We then let $M_{z}$ be the minimizers of $E(n, \cdot)$ over the local set $I_{z}$. Since $E(n, \cdot)$ bounds the distance to the spectrum and converges to the true distance, $M_{z}$ approximates the spectrum near the point $z$.

The negative result (i) we prove shows that it is, in general, impossible to know in finite time if we have computed all of the spectrum up to an arbitrary error parameter. However, the convergence of the algorithm [which depends on the use of the above local search radius $E(n, z)]$ ensures that we will progressively obtain all of the spectrum through this search routine (and avoid solutions not in the bulk spectrum). The algorithm's output $\Gamma_{n}(A)$ is the union of all such $M_{z}$ for $z$ such that $E(n, z) \leq 1 / 2$, and 
the error bound is $E_{n}(A)=\max \left\{E(n, z): z \in \Gamma_{n}(A)\right\}$. The approximate states for $z \in \Gamma_{n}(A)$ then correspond to approximate states of the ground state energy of the folded Hamiltonian $(A-z I)^{*}(A-z I)$, which can be computed efficiently using an $l d l$ decomposition and with residual error bounds. We also give a precise bound on the time needed for the computations based on the size of the region $G_{\mathcal{D}}(n)$, the resolution $\delta(n)^{-1}$, and $n$ (Proposition 4 in Ref. [34]). $\delta(n)$ can also be chosen to vary across $\mathcal{D}$, which we predict will allow extremely fast computation of states corresponding to eigenvalues in gaps of the spectrum.

Results.-We now illustrate our algorithm(s) on difficult models arising in condensed-matter physics or quantum mechanics. In addition, an example considering the nonHermitian Anderson model is included in Ref. [34].

Quasicrystals.-Quasicrystals, and more generally aperiodic systems, have generated considerable interest due to their often exotic physical or spectral properties $[18,19]$. We present the first rigorous spectral computational study with error bounds on a Penrose tile, the standard 2D model of a quasicrystal $[22,43,44]$. No previously known algorithm determines the spectrum with error bounds on the output.

The free Hamiltonian $H_{0}$ (Laplacian) is given by

$$
\left(H_{0} \psi\right)_{i}=\sum_{i \sim j}\left(\psi_{j}-\psi_{i}\right),
$$

with a summation over nearest neighbor sites (vertices). Previous numerical methods study the eigenvalues of the Hamiltonian restricted to a finite portion of the tiling with a choice of boundary conditions at the edges (finite section method) such as periodic approximants [45-49]. This can cause additional eigenvalues (spectral pollution or "edge states") [14] to appear which are not in the spectrum of $H_{0}$ acting on the infinite tiling. While edge states have a physical meaning and are important experimentally, it is often desirable to distinguish these from the "bulk" states of the operator. Our algorithm could have useful applications in the fast-growing research area of topological edge states [50,51].

Figure 1 shows the output of the algorithm for $n=10^{5}$ and two finite section methods (open boundary conditions and periodic approximants). The error estimate, computed (using the algorithm) for both the algorithm and the finite section method, is displayed at each point. This error estimate converges uniformly to the true error on compact subsets of $\mathbb{R}$. Finite section methods produce spurious points in the gaps of the spectrum.

Figure 1 also shows the benefit of parallelization. The time taken for the algorithm (ran using 200 cores) and for the finite section methods (ran using four cores) to reach the final output suggests a speed-up of about 20 times. Moreover, the time for the finite section method appears to grow $\sim \mathcal{O}\left(n^{2.9}\right)$ and $\mathcal{O}\left(n^{3.0}\right)$ for open and periodic boundary conditions, respectively, whereas the time for
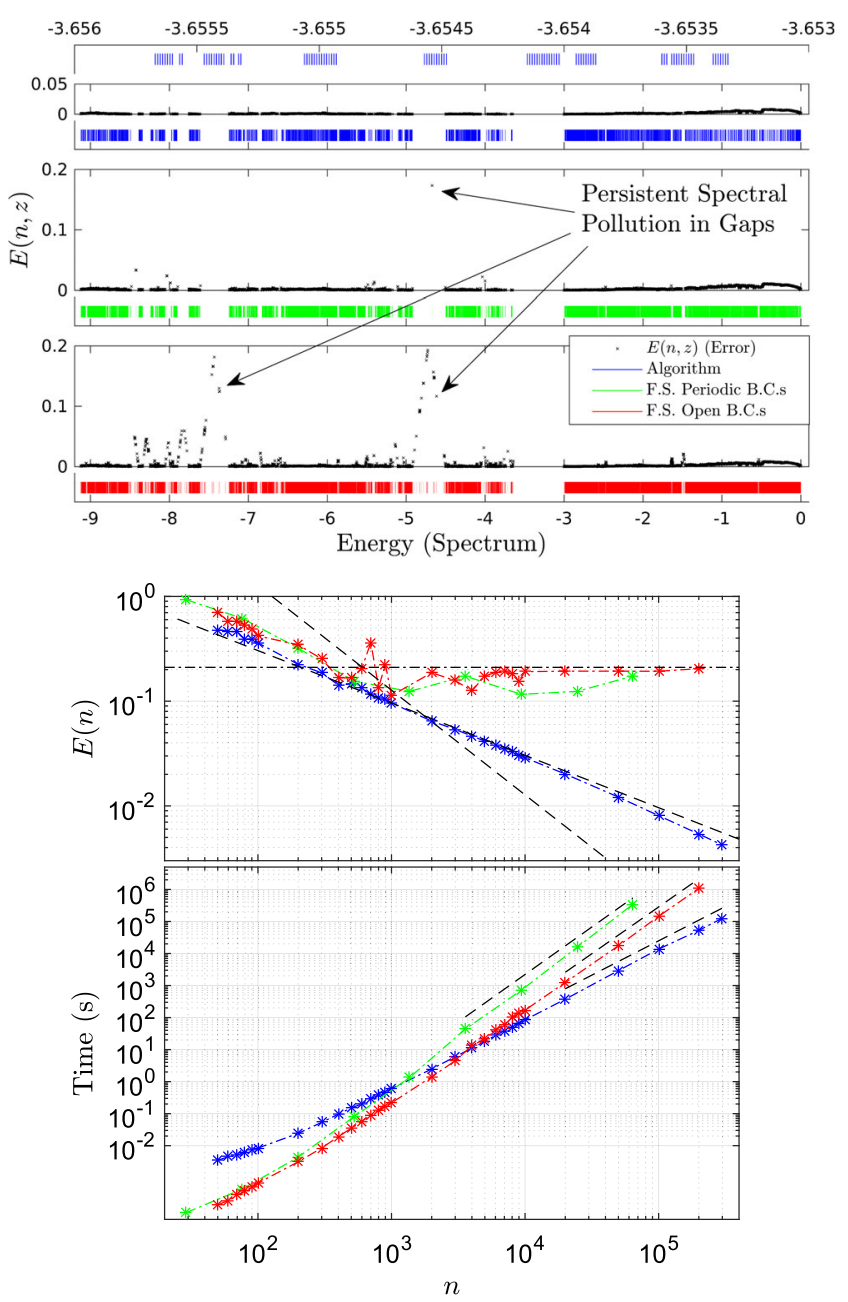

FIG. 1. Top: Large-scale experiment with $n=10^{5}$ for the algorithm and finite section with open boundary conditions and periodic approximants $(n=64079)$. The top shows an enlarged section and the high resolution obtained. The approximation computed with the finite section methods produces spurious points in band gaps with large errors $\sim 0.2$. Bottom: The maximum errors as well as time of outputs for the algorithm (blue) and finite section methods (red for open boundary conditions, green for periodic).

the new algorithm grows $\sim \mathcal{O}\left(n^{2.1}\right)$, predicting larger differences for larger $n$. The direct diagonalization approach is hard to parallelize and so will have difficulty competing with our method for large $n$. It is also possible to use the algorithm to locally compute approximate states corresponding to a given energy level without the need to diagonalize the whole system as shown in Fig. 2.

Finally, we consider a magnetic Hamiltonian [4,52-54]

$$
(H \psi)_{i}=-\sum_{\langle i, j\rangle} e^{i \alpha_{j i}} \psi_{j}
$$

A constant perpendicular magnetic field $\mathbf{B}=B \mathbf{z}$ with potential $\mathbf{A}=(0, x B, 0)$ is applied, leading to the Peierls phase factor between sites $i$ and $j$ : 


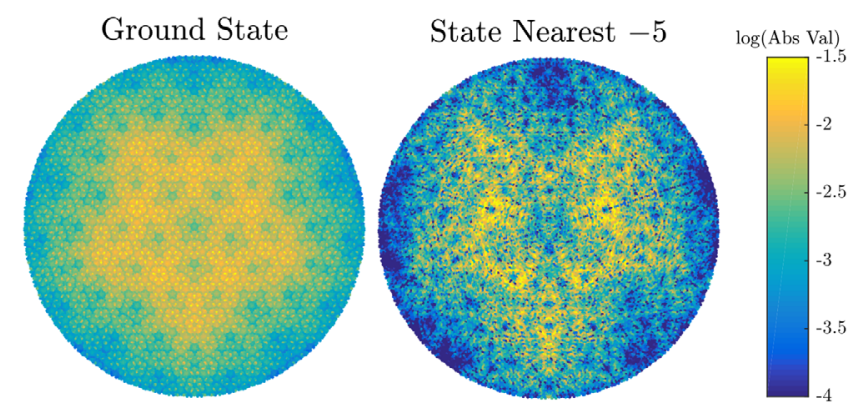

FIG. 2. The ground state for the Penrose Laplacian and a state corresponding to energy nearest -5 . The algorithm allows us to choose which states to compute.

$\alpha_{j i}=\left(2 \pi / \Phi_{0}\right) \int_{\mathbf{r}_{j}}^{\mathbf{r}_{i}} \mathbf{A} \cdot d \mathbf{l}$, where $\Phi_{0}=h c / e$ is the flux quantum. Figure 3 shows the output for the finite section method and the algorithm for $n=5000$ up to the first selfsimilar mode $B_{0}$. The absence of spectral pollution for the new algorithm is striking and agrees well the periodic approximant studied in Ref. [55].

Recently, Hofstadter's butterfly has been experimentally observed in graphene lattices $[20,21,56]$. Clearly, numerical methods that avoid spectral pollution, converge, and provide error bounds are needed to study such operators with fractal-like spectra. Although one may also study this type of problem via periodic approximants as before $[52,55]$, this places restrictions on the value of $B$. One can increase the allowed values by the use of magnetic translations [57], but the total magnetic flux through a period cell must still be a multiple of the flux quantum. Such methods cannot be applied to problems with arbitrary (even nonconstant) magnetic fields nor models with large degrees of freedom, whereas the new algorithm can. Numerical difficulties have previously prevented
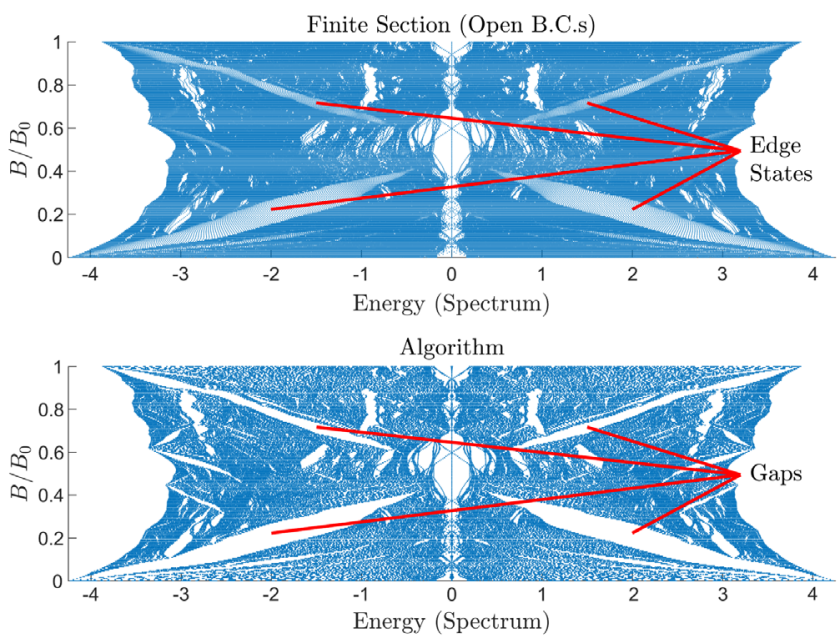

FIG. 3. Comparison of the finite section method and the algorithm for the magnetic Hamiltonian. The algorithm correctly leaves out the gaps and is able to capture the complicated structure with guaranteed error maximum 0.058 for $n=5000$. theoretical modeling of many experimental results of quasicrystals in higher dimensions. The new algorithm can tackle such models, and future work will study 3D systems.

Open systems in optics.-Open systems typically yield non-Hermitian Hamiltonians, as there is no guaranteed energy preservation. However, non-Hermitian Hamiltonians can possess real spectra when they respect parity-time $(P T)$ symmetry [58-60]. Remarkably, many Hamiltonians undergo a phase transition to complex spectra if the imaginary part of their potential is increased beyond a certain threshold, known as symmetry breaking. Such systems are of wide interest [61-65] and can be realized in optics [25-29,66-69].

Detecting when symmetry breaking occurs poses a substantial challenge, since it is very sensitive to surface
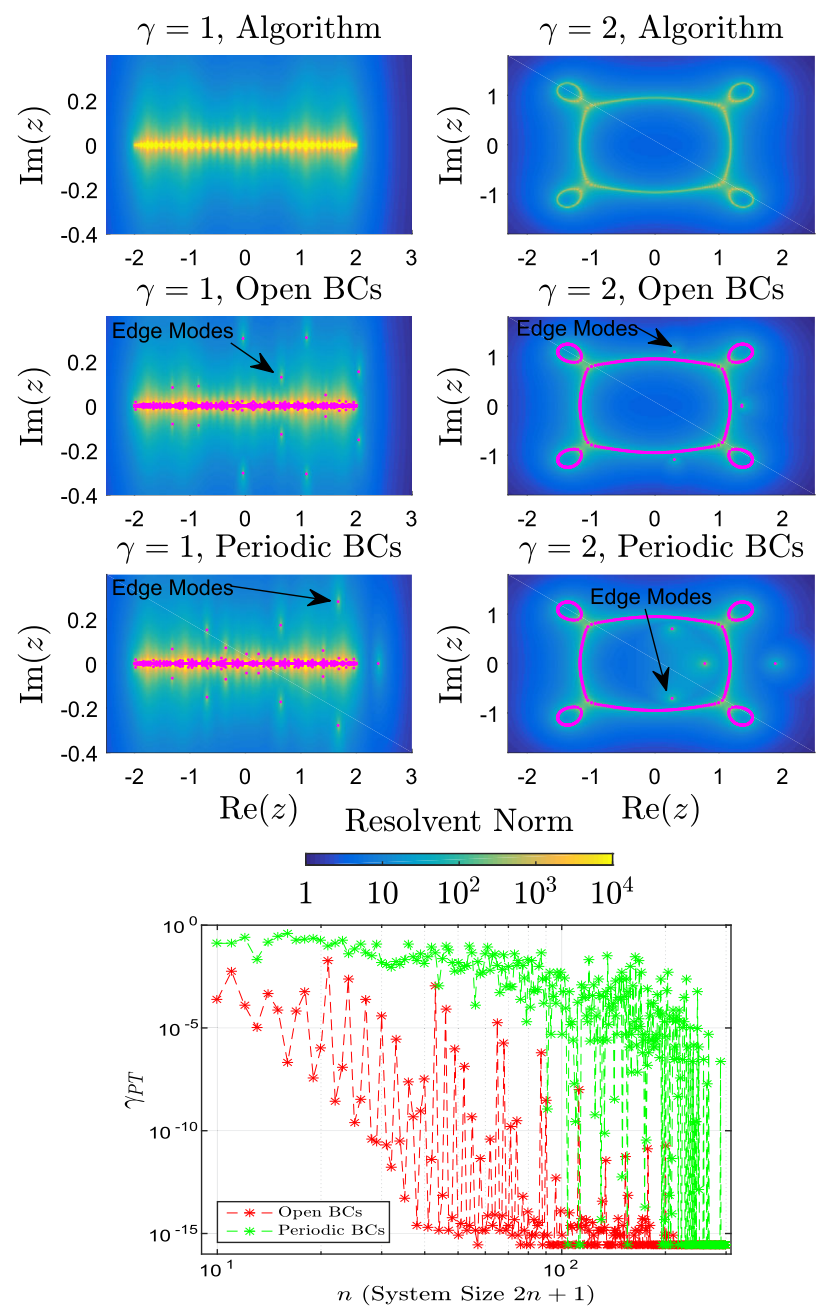

FIG. 4. Top: Pseudospectra of $H$ computed with the proposed algorithm and finite sections (spectrum in magenta). Bottom: Fragile $P T$-symmetric phase as we increase the system size due to edge states (caused by spectral pollution) with complex eigenvalues, verifying the unsuitability of algorithms based on finite sections. 
states arising from standard truncations. We discuss $P T$ symmetry breaking for the case of an aperiodic potential on a discrete lattice:

$$
(H x)_{n}=x_{n-1}+x_{n+1}+V_{n} x_{n}
$$

acting on $l^{2}(\mathbb{Z})$, where $V_{n}=\cos (n)+i \gamma \sin (n)$ and $\gamma \geq 0$. Here the aperiodicity occurs dues to the incommensurability of the potential and lattice. We stress that the algorithm can handle any type of potential (such as additional defects).

In the limit of an increasing system size, the critical parameter $\gamma_{P T}$ depends on the boundary conditions imposed, often decreasing as the number of sites increases with a fragile $P T$-symmetric phase. This limit can differ from the value $\gamma_{P T}$ on the infinite lattice due to surface or edge states [70]. Using the algorithm gives an estimate for $\gamma_{P T}$ in the infinite lattice case avoiding this fragility, suggesting that symmetry breaking occurs at $\gamma_{P T} \approx 1 \pm 0.05$. This allows us to rigorously detect edge states (corresponding to spectral pollution) and the corresponding edge modes. Figure 4 shows pseudospectral plots generated by the algorithm for $\gamma=1,2$ as well as the corresponding plots for finite chains of length 2001 for open and periodic boundary conditions. We can easily use the algorithm to separate bulk states from edge states. We have also shown the values of $\gamma_{P T}$ for the finite chains showing the fragility of the $P T$-symmetric phase.

Conclusion.-We have demonstrated the boundaries of what computers can do in spectral computations in large areas of applications by presenting the first algorithms that converge to the spectrum or pseudospectrum with error control on the output. In contrast, the state of the art finite section method, even for the cases where it does converge, does not provide error bounds. The related issue of spectral pollution has been well studied with previous results concentrating on the self-adjoint case [71], in particular, detecting spectral pollution in gaps of the essential spectrum. However, none of these methods provides convergence to the spectrum, as shown by Shargorodsky in Ref. [72]. The major difference between the algorithms presented here and those previously studied is the local approximation of the resolvent norm using uneven sections. Physically, for discrete systems, this corresponds to preserving the correct interactions of the first $n$ sites and avoiding the use of imposed boundary conditions.

The presented method is very general and can be used in many diverse fields of applications. A significant advantage is the possibility of error control that will allow scientists to compare experiments with computational results that are now guaranteed to be correct up to an accuracy parameter set by the user. Moreover, the locality of the algorithms represents a considerable advantage if one is interested only in certain regions of the spectrum and makes computations extremely quick.
This work was supported by Engineering and Physical Sciences Research Council Grants No. EP/L016516/1, No. EP/R008272/1, No. EP/N014588/1, and No. EP/ L003457/1, as well as a Royal Society University Research Fellowship.

[1] E. Schrödinger, in Proceedings of the Royal Irish Academy. Section A: Mathematical and Physical Sciences (JSTOR, Dublin, 1940), Vol. 46, pp. 9-16.

[2] P. W. Anderson, Phys. Rev. 109, 1492 (1958).

[3] E. Balslev and J.-M. Combes, Commun. Math. Phys. 22, 280 (1971).

[4] D. R. Hofstadter, Phys. Rev. B 14, 2239 (1976).

[5] R. Haydock, Comput. Phys. Commun. 20, 11 (1980).

[6] L. E. Gendenshtein, JETP Lett. 38, 356 (1983).

[7] S. Ostlund, R. Pandit, D. Rand, H. J. Schellnhuber, and E. D. Siggia, Phys. Rev. Lett. 50, 1873 (1983).

[8] A. Sütő, Commun. Math. Phys. 111, 409 (1987).

[9] N. Laskin, Phys. Rev. E 66, 056108 (2002).

[10] A. Böttcher and B. Silbermann, Introduction to Large Truncated Toeplitz Matrices, Universitext (Springer, New York, 2012).

[11] A. Böttcher, J. Integr. Equations Appl. 6, 267 (1994).

[12] T. Digernes, V. S. Varadarajan, and S. R. S. Varadhan, Rev. Math. Phys. 06, 621 (1994).

[13] A. C. Hansen, J. Am. Math. Soc. 24, 81 (2011).

[14] M. Marletta, IMA J. Numer. Anal. 30, 917 (2010).

[15] L. N. Trefethen and M. Embree, Spectra and Pseudospectra: The Behavior of Nonnormal Matrices and Operators (Princeton University, Princeton, NJ, 2005).

[16] E. B. Davies, Linear Operators and Their Spectra (Cambridge University Press, Cambridge, England, 2007), Vol. 106.

[17] W. Arveson, in $C^{*}$-Algebras: 1943-1993 (San Antonio, TX, 1993), Contemporary Mathematics Vol. 167 (American Mathematical Society, Providence, RI, 1994), pp. 114-129.

[18] D. Shechtman, I. Blech, D. Gratias, and J. W. Cahn, Phys. Rev. Lett. 53, 1951 (1984).

[19] Z. M. Stadnik, Physical Properties of Quasicrystals (Springer Science, New York, 2012), Vol. 126.

[20] B. Hunt, J. Sanchez-Yamagishi, A. Young, M. Yankowitz, B. J. LeRoy, K. Watanabe, T. Taniguchi, P. Moon, M. Koshino, P. Jarillo-Herrero et al., Science 340, 1427 (2013).

[21] C. Dean, L. Wang, P. Maher, C. Forsythe, F. Ghahari, Y. Gao, J. Katoch, M. Ishigami, P. Moon, M. Koshino et al., Nature (London) 497, 598 (2013).

[22] Z. V. Vardeny, A. Nahata, and A. Agrawal, Nat. Photonics 7, 177 (2013).

[23] L. Levi, M. Rechtsman, B. Freedman, T. Schwartz, O. Manela, and M. Segev, Science 332, 1541 (2011).

[24] A. Sütő, J. Stat. Phys. 56, 525 (1989).

[25] A. Regensburger, C. Bersch, M.-A. Miri, G. Onishchukov, D. N. Christodoulides, and U. Peschel, Nature (London) 488, 167 (2012).

[26] A. Guo, G. Salamo, D. Duchesne, R. Morandotti, M. Volatier-Ravat, V. Aimez, G. Siviloglou, and D. Christodoulides, Phys. Rev. Lett. 103, 093902 (2009). 
[27] C. E. Rüter, K. G. Makris, R. El-Ganainy, D. N. Christodoulides, M. Segev, and D. Kip, Nat. Phys. 6, 192 (2010).

[28] L. Feng, Z. J. Wong, R.-M. Ma, Y. Wang, and X. Zhang, Science 346, 972 (2014).

[29] H. Hodaei, M.-A. Miri, M. Heinrich, D. N. Christodoulides, and M. Khajavikhan, Science 346, 975 (2014).

[30] T. S. Cubitt, D. Perez-Garcia, and M. M. Wolf, Nature (London) 528, 207 (2015).

[31] T. D. Schultz, D. C. Mattis, and E. H. Lieb, Rev. Mod. Phys. 36, 856 (1964).

[32] M. B. Hastings, Phys. Rev. B 69, 104431 (2004).

[33] I. Affleck, T. Kennedy, E. H. Lieb, and H. Tasaki, in Condensed Matter Physics and Exactly Soluble Models (Springer, New York, 1988), pp. 253-304.

[34] See Supplemental Material at http://link.aps.org/ supplemental/10.1103/PhysRevLett.122.250201 for further details, including proofs of results, algorithmic detail, and remarks on efficient numerical implementation as well as implications in quantum mechanics and Refs. [35-41].

[35] A. M. Turing, Proc. London Math. Soc. s2-42, 230 (1937).

[36] L. Blum, F. Cucker, M. Shub, and S. Smale, Complexity and Real Computation (Springer-Verlag, New York, 1998).

[37] L. N. Trefethen and D. Bau III, Numerical Linear Algebra (Society for Industrial and Applied Mathematics, Philadelphia, 1997), Vol. 50.

[38] N. Hatano and D. R. Nelson, Phys. Rev. Lett. 77, 570 (1996).

[39] S. Longhi, D. Gatti, and G. Della Valle, Sci. Rep. 5 (2015).

[40] D. R. Nelson and N. M. Shnerb, Phys. Rev. E 58, 1383 (1998).

[41] I. Y. Goldsheid and B. A. Khoruzhenko, Phys. Rev. Lett. 80, 2897 (1998).

[42] D. Krejčiřík, P. Siegl, M. Tater, and J. Viola, J. Math. Phys. (N.Y.) 56, 103513 (2015).

[43] S. Takemura, N. Takemori, and A. Koga, Phys. Rev. B 91, 165114 (2015).

[44] A. Della Villa, S. Enoch, G. Tayeb, V. Pierro, V. Galdi, and F. Capolino, Phys. Rev. Lett. 94, 183903 (2005).

[45] H. Tsunetsugu, T. Fujiwara, K. Ueda, and T. Tokihiro, Phys. Rev. B 43, 8879 (1991).

[46] M. A. Bandres, M. C. Rechtsman, and M. Segev, Phys. Rev. X 6, 011016 (2016).

[47] E. C. Andrade, A. Jagannathan, E. Miranda, M. Vojta, and V. Dobrosavljević, Phys. Rev. Lett. 115, 036403 (2015).

[48] A. Szallas and A. Jagannathan, Phys. Rev. B 77, 104427 (2008).
[49] N. de Bruijn, Indagationes Math. (Proceedings) 84, 39 (1981).

[50] M. Z. Hasan and C. L. Kane, Rev. Mod. Phys. 82, 3045 (2010).

[51] X.-L. Qi and S.-C. Zhang, Rev. Mod. Phys. 83, 1057 (2011).

[52] D.-T. Tran, A. Dauphin, N. Goldman, and P. Gaspard, Phys. Rev. B 91, 085125 (2015).

[53] T. Hatakeyama and H. Kamimura, Solid State Commun. 62, 79 (1987).

[54] J. Vidal and R. Mosseri, J. Non-Cryst. Solids 334-335, 130 (2004).

[55] T. Hatakeyama and H. Kamimura, J. Phys. Soc. Jpn. 58, 260 (1989).

[56] L. Ponomarenko, R. Gorbachev, G. Yu, D. Elias, R. Jalil, A. Patel, A. Mishchenko, A. Mayorov, C. Woods, J. Wallbank et al., Nature (London) 497, 594 (2013).

[57] J.-N. Fuchs and J. Vidal, Phys. Rev. B 94, 205437 (2016).

[58] C. M. Bender and S. Boettcher, Phys. Rev. Lett. 80, 5243 (1998).

[59] S. Klaiman, U. Günther, and N. Moiseyev, Phys. Rev. Lett. 101, 080402 (2008).

[60] C. M. Bender, Rep. Prog. Phys. 70, 947 (2007).

[61] T. Gao, E. Estrecho, K. Bliokh, T. Liew, M. Fraser, S. Brodbeck, M. Kamp, C. Schneider, S. Höfling, Y. Yamamoto et al., Nature (London) 526, 554 (2015).

[62] M. Wimmer, A. Regensburger, M.-A. Miri, C. Bersch, D. N. Christodoulides, and U. Peschel, Nat. Commun. 6, 77782 (2015).

[63] T. Eichelkraut, R. Heilmann, S. Weimann, S. Stützer, F. Dreisow, D. Christodoulides, S. Nolte, and A. Szameit, Nat. Commun. 4 (2013).

[64] H. Schomerus, Opt. Lett. 38, 1912 (2013).

[65] S. Longhi, Phys. Rev. Lett. 103, 123601 (2009).

[66] K. G. Makris, R. El-Ganainy, D. N. Christodoulides, and Z. H. Musslimani, Phys. Rev. Lett. 100, 103904 (2008).

[67] J. Schindler, A. Li, M. C. Zheng, F. M. Ellis, and T. Kottos, Phys. Rev. A 84, 040101 (2011).

[68] A. Regensburger, M.-A. Miri, C. Bersch, J. Näger, G. Onishchukov, D. N. Christodoulides, and U. Peschel, Phys. Rev. Lett. 110, 223902 (2013).

[69] B. Zhen, C. W. Hsu, Y. Igarashi, L. Lu, I. Kaminer, A. Pick, S.-L. Chua, J. D. Joannopoulos, and M. Soljačić, Nature (London) 525, 354 (2015).

[70] O. Bendix, R. Fleischmann, T. Kottos, and B. Shapiro, Phys. Rev. Lett. 103, 030402 (2009).

[71] E. B. Davies, LMS J. Comput. Math. 1, 42 (1998).

[72] E. Shargorodsky, J. Spectr. Theory 3, 535 (2013). 